R.J. Marks II, J.F. Walkup and M.O. Hagler "Volume hologram representation of spacevariant systems", in Applications of Holography and Optical Data Processing edited by E. Marom, A.A. Friesem and E. Wiener-Aunear, Oxford: Pergamon Press, pp.105-113 (1977). 


\title{
VOLUME HOLOGRAM REPRESENTATION OF SPACE-VARIANT SYSTEMS
}

\author{
Robert J. Marks II, John F. Walkup and Marion O. Hagler \\ Department of Electrical Engineering, Texas Tech University, Lubbock, Texas 79409 \\ ABSTRACT
}

Methods of characterizing linear space-variant systems by their responses to various sets of inputs are discussed. Recording these responses within a volume hologram results in a filter which is approximately equivalent, in an input-output sense, to the space-variant system.

The approaches considered are (1) storing the transfer functions of the system for point sampling of the input plane on playback: (2) a piecewise isoplanatic approximation approach based on division of the input plane into isoplanatic regions, with storage of the transfer function for each isoplanatic patch; and (3) storage of the system's responses to elements of an orthonormal basis set. The potential advantages and limitations of each of these approaches, as well as experimental results, are discussed.

\section{INTRODUCTION}

It is well known that a holographically recorded filter can be placed in the Fourier plane of a coherent optical processor and used to represent the transfer function of a linear, space-invariant system. This single filter displays the input-output characteristics of the corresponding system (1). Unfortunately, many optical systems which one might desire to represent holographically are space-variant. For example, even an ideal imaging system with non-unity magnification is rigorously space-variant. Space-variant systems may, however, be characterized by cataloging the system's responses to a number of separate inputs, as contrasted with the use of the single point spread function required to characterize the space-invariant system. If a thick medium is used to store the system responses, the resulting volume hologram can subsequently act as a space variant filter which exhibits the input-output characteristics of the original system. Such a representation can, in principle, significantly reduce the weight and size of a coherent processor, and should also improve its orientation stability.

For mathematical and notational simplicity attention will be restricted to one dimensional linear, space-variant systems. For additional details, the reader is referred to some recent papers by the authors and their colleagues (2-6). Space variant systems, 
characterized by the linear operator $s[\cdot]$, may be described by the superposition integral

$$
g(x)=S[f(x)]=\int_{\infty}^{\infty} f(\xi) h(x-\xi ; \xi) d \xi
$$

where $f(x)$ is the input, $g(x)$ is the output, and $h(x-\xi ; \xi)$ represents the space-variant line spread function. That is

$$
\mathrm{h}(\mathrm{x}-\xi ; \xi)=\mathrm{s}[\delta(\mathrm{x}-\xi)]
$$

where $\delta(x)$ is the Dirac delta function. This form of the line spread function has some advantages in describing space-variant systems $(6-7)$. In the event that $S$ is space invariant, we find that

$$
h(x-\xi ; \xi) \rightarrow h(x-\xi)
$$

which says that $h(x-\xi ; \xi)$ is independent of its second argument.

We now consider three approaches to representing the effects of the space-variant system S described by Eq. (1). Each of these approaches may, at least in principle, be implemented holographically. Some details on the holographic implementation of the sampling theorem approach are given in a later section.

\section{THE SAMPLING THEOREM APPROACH}

A modification of the Whittaker-Shannon sampling theorem (1) permits us to design a holographic representation for space-variant systems based on line source sampling of the input plane of $S$, and subsequent angle-multiplexed holographic storage of the transfer function of $\mathrm{S}$ for each input plane sample.

The sampling theorem for space-variant systems (5) is based on the concept of the system's variation spectrum, which is defined by

$$
\mathrm{H}_{\xi}(\mathrm{x} ; \mathrm{v}) \triangleq \mathrm{F}_{\xi}[\mathrm{h}(\mathrm{x} ; \xi)]
$$

where $F_{\xi}[\cdot]$ denotes Fourier transformation with respect to the input variable $\xi$, and where $\mathrm{v}$ is the frequency variable associated with $\xi$. The variation spectrum is a measure of how the line spread function changes form with respect to the input variable $\xi$. In brief, the theorem states that if the input, $f(\xi)$, is bandlimited to bandwidth $2 \mathrm{~W}_{f}$, and if $\mathrm{h}(\mathrm{x} ; \xi)$ has a variation spectrum of width $2 w_{v}$ (i.e. $H_{\xi}(x ; v)=0$ for $|v|>w_{v}$ for all $\left.x\right)$, then their product $f(\xi) h(x ; \xi)$ will have bandwidth $2 \mathrm{~W}=2 \mathrm{~W}_{f}+2 \mathrm{~W}_{\mathrm{v}}$. As a result, the sampling theorem states that by sampling the input line to the system $S$ at a rate of $2 \mathrm{~W}$ samples per unit length, the output $g(x)$ may be written as the infinite sum

$$
g(x)=\sum_{n=-\infty}^{\infty} f\left(\xi_{n}\right) h\left(x-\xi_{n} ; \xi_{n}\right) * \operatorname{sinc}(2 W x)
$$

where $\xi_{n}=n / 2 W$. Equivalently, in the frequency domain, 


$$
G\left(f_{x}\right)=\frac{1}{2 W} \sum_{n=-\infty}^{\infty} f\left(\xi_{n}\right) H_{x}\left(f_{x} ; \xi_{n}\right) \exp \left(-j 2 \pi f_{x} \xi_{n}\right) \operatorname{rect}\left(f_{x} / 2 W\right)
$$

where, $\operatorname{sinc} x=\frac{\sin \pi x}{\pi x}$, and

$$
\operatorname{rect} x \triangleq \begin{aligned}
& 1,|x|<\frac{1}{2} \\
& 0,|x| \geq \frac{3}{2}
\end{aligned}
$$

Equation (6) states that $G\left(f_{x}\right)$ may be obtained as a weighted sum of individual transfer functions $\mathrm{H}_{x}\left(f_{x} ; \xi_{n}\right)$, where the $n{ }^{\text {th }}$ of which is weighted by the $n^{\text {th }}$ input sample $f\left(\xi_{n}\right)$. The presence of rect $\left(f_{x} / 2 W\right)$ in Eq. (6) indicates the low pass interpolation filter (bandwidth 2W) function necessary to reconstruct the continuous output $\mathrm{g}(\mathrm{x})$. One obvious problem with Eqs. (5) and (6) is that they require us to store a countably infinite number of holograms for an exact reconstruction of $\mathrm{g}(\mathrm{x})$. In practice we would expect to approximate $g(x)$ by storing a finite number determined by space-bandwidth product considerations.

The advantage of the sampling theorem approach is that it specifies a technique for exact reconstruction of the continuous output $g(x)$ (i.e. low pass filtering). A disadvantage is that it.requires that we sample the input to $S$ at a minimum rate determined by the sum of the variation bandwidth of $S$ and the input bandwidth. One technique for cutting down on the density of input plane samples required is to employ the piecewise isoplanatic approximation (PIA) approach described next.

\section{THE PIECEWISE ISOPLANATIC APPROXIMATION (PIA) APPROACH}

The piecewise isoplanatic approximation, or PIA approach (4) makes the assumption that the space-variant system $\mathrm{S}$ is piecewise spaceinvariant (see Ref. I). It effectively divides the input line into segments (or the plane into "patches"), and each segment is characterized by its own line spread function. Mathematically we may rewrite the input $f(\xi)$ as

$$
\begin{aligned}
f(\xi) & =\sum_{m} f_{m}\left(\xi-\xi_{m}\right) \\
& =\sum_{m} f(\xi) \operatorname{rect}\left(\xi ; \ell_{m^{\prime}} u_{m}\right)
\end{aligned}
$$

where rect $\left(\xi ; l_{m}, u_{m}\right)$ represents a rectangle function of unit height, extending from $\xi=\ell_{m}$ to $\xi=u_{m^{\prime}}$ and $\ell_{m} \leq \xi_{m} \leq u_{m}$. Since $g(x)=s[f(\xi)]$ we obtain

$$
g(x)=\sum_{m} s\left[f_{m}\left(\xi-\xi_{m}\right)\right]
$$

or equivalently 


$$
g(x)=\sum_{m} \int_{l_{m}}^{u_{m}} f(\xi) h\left(x-\xi ; \xi_{m}\right) d \xi
$$

When compared with the sampling theorem approach, the PIA approach appears to offer the advantage of being independent of the bandwidth of the input function $f(x)$. Rather it is the manner in which the line spread function $h(x-\xi ; \xi)$ changes which determines the number of holograms which must be stored to represent the system. Depending on the relative sizes of the system's variation bandwidth and the input bandwidth, this advantage may or may not be significant. It would appear to be difficult, in general, to compare the performance of a PIA-based implementation with one based on the sampling theorem in situations where systems are not truly piecewise isoplanatic.

In the next section we discuss the orthonormal response approach, where the nature of the input function must be considered, but where the nature of the space-variant system is in general, not a determining factor.

\section{THE ORTHONORMAL RESPONSE APPROACH}

A third approach to characterizing the space-variant system is to expand the input function $f(x)$ as a weighted sum of the elements of an orthonormal basis set. Thus we write

$$
f(x)=\sum_{n} \alpha_{n} \phi_{n}(x)
$$

where the elements of the set $\left\{\phi_{n}(x): n=1,2, \ldots\right\}$ are assumed orthonormal, i.e.

$$
\int_{-\infty}^{\infty} \phi_{n}(\xi) \phi_{m}^{*}(\xi) d \xi=\delta_{n m}
$$

with $\delta_{n m}$ being the familiar Kronecker delta function. Substituting Eq. (11) into the relationship $g(x)=s[f(x)]$ we obtain

$$
g(x)=\sum_{n} \alpha_{n} s\left[\phi_{n}(x)\right]
$$

where the expansion coefficients are found by

$$
\alpha_{n}=\int_{-\infty}^{\infty} f(\xi) \phi_{n}^{*}(\xi) d \xi
$$

Note now that if we let $\Phi_{n}(x)$ be the response of $S$ to the input $\phi_{n}(x)$,

$$
\Phi_{\mathrm{n}}(\mathrm{x})=\mathrm{S}\left[\Phi_{\mathrm{n}}(\mathrm{x})\right]
$$

then Eq. (13) may be rewritten

$$
g(x)=\sum_{n} \alpha_{n} \Phi_{n}(x)
$$


It is worth noting here that the elements of the system response set $\left\{\Phi_{n}(x)\right\}$ are not necessarily orthonormal, as are the elements of the input set $\left\{\phi_{n}(x)\right\}$.

To illustrate this approach, if we assume that $f(x)$ is bandlimited to bandwidth $2 \mathrm{~W}_{f}$, then by the sampling theorem

$$
f(x)=\sum_{n=-\infty}^{\infty} f\left(x_{n}\right) \text { sinc } 2 W_{f}\left(x-x_{n}\right)
$$

where $x_{n}=n / 2 w_{f}$. We can view Eq. (17) as an orthonormal expansion with

$$
\phi_{n}(x)=\sqrt{2 W_{f}} \operatorname{sinc} 2 w_{f}\left(x-x_{n}\right)
$$

and with

$$
\alpha_{n}=\frac{1}{\sqrt{2 W_{f}}} f\left(x_{n}\right)
$$

The system's sinc response is then given by

$$
\operatorname{s}\left[\operatorname{sinc} 2 \mathrm{~W}_{\mathrm{f}}\left(\mathrm{x}-\mathrm{x}_{\mathrm{n}}\right)\right]=\frac{1}{\sqrt{2 \mathrm{~W}_{\mathrm{f}}}} \Phi_{\mathrm{n}}(\mathrm{x})
$$

and the system output, $g(x)$, may be written as

$$
g(x)=\sum_{n} f\left(x_{n}\right) \text { s }\left[\operatorname{sinc} 2 w_{f}\left(x-x_{n}\right)\right]
$$

Note here that the advantage of the orthonormal response characterization is that only the input bandwidth determines the minimum required input plane sampling rate, not the variation bandwidth of $S$. In addition, since the sinc function is just the Fourier transform of the rectangle function (Ref. 1), physical generation of a coherent system's sinc response is easily implemented. A possible approach to implementing the sinc response approach is discussed at the end of the next section.

We have presented three approaches to characterizing the performance of space-variant systems. In principle, each of these approaches can be implemented experimentally. In our experimental work to date we have assumed point sampling of the input plane of a system, with the idea of implementing the sampling theorem approach. In the next section we briefly discuss some of the practical limitations present when one attempts to angle multiplex the sampled transfer functions of a space-variant system within a thick recording medium.

\section{IMPLEMENTATIONS}

Our experimental work to date has concentrated on the holographic implementation of the sampling theorem approach (2-3). Figure 1 illustrates (in one dimension) the basic approach. Point sources are used to sample the input plane of the space-variant system $S$, 
resulting in the system's point spread function appearing at the output of $\mathrm{S}$. The $\mathrm{n}^{\text {th }}$ reference point source, shown offset by $a$, also lies in the output plane of $\mathrm{S}$. After the lens $L$ performs $a$ Fourier transform, the interference of the reference plane wave and the transfer function of $S$ for the $n^{\text {th }}$ point source input is holographically recorded in the thick medium. When we perform this operation sequentially, with a different reference point source for each object point source, we are angle multiplexing a number of transfer function holograms into the medium. By using the extinction angle concept we can guarantee essentially noninterfering holograms.

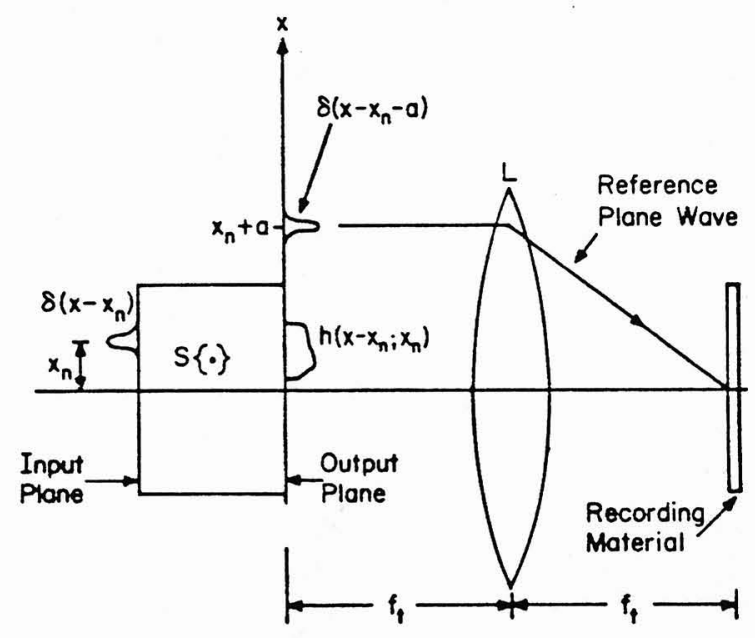

Fig. 1. Recording volume hologram: sampling theorem approach

One places the volume hologram in the Fourier plane of a coherent optical processor for playback, as indicated schematically in Fig. 2. On playback one spatially samples the input plane using a duplicate of the reference array. Each input point accesses the hologram which represents the transfer function of $S$ for that input point location. Neglecting crosstalk between the stored holograms, coherent addition of the outputs then gives the desired response. The experiments have mainly been performed using a Dupont holographic photopolymer (8) as the recording material, and an Argon laser operating at $5145 \AA$ (2-3). Experimental implementations of simple one- and two-lens magnifiers for objects consisting of simple arrays of point sources have been produced and found to yield the correct magnifications though the images contained some aberrations. Additional experimental work is in progress. 
The extinction angle, $\Delta \theta$, is the major factor affecting system resolution $(2-3,9)$. One can show that even in the worst case where the system point spread functions associated with sampling the input plane of $S$ overlap each other completely, it is possible, by properly spacing the reference array point sources, to obtain noninterfering holograms in the thick recording medium. If we assume (see Fig. 1) that the transforming lens has focal length $f_{t}>>a$, the reference array offset, we find that based on the assumption that the extinction angle is essentially invariant as one moves the reference and object beams over their respective arrays (a reasonable approximation in many cases), the minimum reference array element-to-element spacing is given by

$$
\Delta x_{\min } \cong f_{t} \Delta \theta
$$

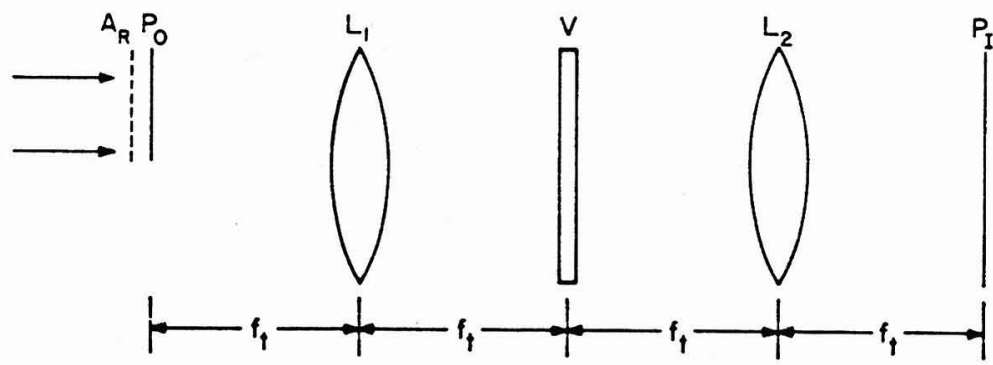

Fig. 2. Playback scheme: sampling theorem approach

This spacing will assure minimum hologram crosstalk on playback. To illustrate, we found that for a 100 micron thick layer of the Dupont holographic photopolymer, with $f_{t}=10 \mathrm{~cm}$. and with an on axis object array, plus a $30^{\circ}$ reference array offset, the extinction angle was $\Delta \theta \tilde{=} 2^{\circ}$. Based on $\mathrm{Eq}$. (22) this predicts that $\Delta \mathrm{x}_{\mathrm{min}}$ $=3.5 \mathrm{~mm}$. Since on playback one illuminates the input transparency through a duplicate of the reference array, the maximum spatial frequency present in the object for which we could achieve the Nyquist sampling rate would be $1 / 2 \Delta \mathrm{x}_{\mathrm{min}}=0.14$ cycles per millimeter $=3.6$ lines per inch. While this is not a very high resolution, it should also be noted that thick recording media such as photochromic glasses exist with extinction angles one and two orders of magnitude smaller than the example just cited (10), so that the state of the art predicts much higher system capabilities. It is clear, however, that storing large numbers of well require, poses numerous problems. These include signal-to noise ratio problems, crosstalk problems, and dynamic range problems, to mention just a few. An additional problem involves the difficulty in obtaining resolutions, for two dimensional representations, which are equal to the one dimensional system resolutions predicted on the basis of the extinction angle concept (11). 
A comment should be made concerning implementation of the sinc response approach discussed in Eqs. (17)-(21). Based on Eq. (21) and Figs. 1-2, we see that by replacing the Dirac delta function by a sinc function at the input to $s$, one can effectively use the same recording geometry for implementing the sinc response approach as was used in implementing the sampling theorem approach.

\section{SUMMARY}

The three approaches presented for synthesizing and using volume holograms to represent space-variant optical systems appear promising, despite some obvious practical limitations. The potential savings offered by such holographic optical systems representations should, however, be sufficient to motivate further research into overcoming some of the problems identified to date. Additional work is underway at present to explore the potential and limitations of these and alternative techniques for optically representing space-variant optical processors.

\section{ACKNOWLEDGEMENTS}

The authors wish to express their appreciation to Dr. Thomas F. Krile of the Rose-Hulman Institute of Technology, Terre Haute, Indiana, for his suggestions during the course of the research. This work has been supported by the Air Force office of Scientific Research, USAF, under Grant AFOSR-75-2855A.

\section{REFERENCES}

(1) J. W. Goodman, Introduction to Fourier Optics, McGraw-Hill, New York, 1968.

(2) L. M. Deen, J. F. Walkup and M. O. Hagler, Representations of space-variant optical systems using volume holograms, Appl. Opt., 14, 2438 (1975).

(3) J. F. Walkup and M. O. Hagler, Volume hologram representations of space-variant optical systems, Proc. of Tech. Prgrm., Electro-Optical Systems Design Conf.-1975, Anaheim, Calif., Nov. 11-13, 1975, pp. 31-37.

(4) R. J. Marks II and T. F. Krile, Systems theory for holographic representation of space-variant systems, Appl. Opt. (to appear) .

(5) R. J. Marks II, J. F. Walkup and M. O. Hagler, A sampling theorem for space-variant systems, J. Opt. Soc. Am., 66 (1976to appear).

(6) R. J. Marks II, J. F. Walkup and M. O. Hagler, on line spread function notation, Appl. Opt., 15 (1976-to appear).

(7) A. W. Lohmann and D. P. Paris, Space-variant image formation, 
J. Opt. Soc. Am., 55, 1007 (1965).

(8) B. L. Booth, Photopolymer material for holography, Appl. Opt., 14,593 (1975).

(9) H. M. Smith, Principles of Holography, 2nd edition, WileyInterscience, New York, 1975.

(10) A. A. Friesem and J. I. Walker, Thick absorption recording media in holography, Appl. Opt., 9, 201 (1970).

(11) R. J. Collier, C. B. Burckhardt and I. H. Lin, Optical Holography, Academic Press, New York, 1971. 